\title{
A Coutourlet-Based Image Watermarking Us- ing Generalized Gaussian Distribution Model
}

\author{
Yin-fang Zhu' \\ Network and Educational Technology Center yichun university yichun \\ 336000 China
}

\begin{abstract}
With the increasing demands of copyright protection, digital watermarking has being paid more and more attention. In the design of a watermarking method, the modelling of signal by a general parametric family of statistical distributions plays an important role in many signal processing applications. Some conventional methods based on Gaussian distribution to model the image coefficients in the transform domain. In this paper, I proposed to adopt the generalized Gaussian distribution (GGD) for modelling the contourlet transform sub-band coefficients and for image watermarking scheme. The contourlet transform (perfect reconstruction and directional selectivity) is considered. Its improved robustness and imperceptibility are due to embedding in the directional subband with the highest energy. In watermark detection, the Neyman-Pearson (NP) detector is used to detect the watermark. Experimental results show that the effectiveness of the presented watermarking method and its robustness against common image processing and some kinds of geometric attacks.
\end{abstract}

Keywords: Digital Watermarking; Generalized Gaussian distribution; Contourlet transform.

\subsection{Introduction}

With the rapid development of computer technologies, it has improved the ease of access to digital multimedia with the rapid development of information technologies, and it also leads to the problem of illegal copying and redistribution of mul-

\footnotetext{
${ }^{1}$ Yin Fang Zhu

Network and Educational Technology Center yichun university yichun 336000 China

Email:ycu3201515@163.com
} 
timedia in the internet ${ }^{[1-2]}$. So it needs novel technologies for the purpose of copyright protection, data authentication and so forth. Digital watermarking is one of the technologies of copyright protection.

It is well known that current methods for the watermarking of images can be divided into two classifications; one is the spatial domain methods and the other is the transform domain methods. Such as the Discrete Fourier Transform (DFT) ${ }^{[3]}$, Discrete Cosine Transform (DCT) ${ }^{[4]}$,Discrete Wavelet Transform (DWT) ${ }^{[5]}$ et al. It is well known that the watermarking strategy based on transform domain has been demonstrated to be more robust against most of attacks ${ }^{[6]}$.

For watermark detection, it has been known that the correlation detector is suboptimal for watermarking in the transform domain, several alternative optimum and locally optimum decoders have been presented ${ }^{[4][7]}$.In [4] and [7], a robust optimum detector using the DCT and DWT. The distribution of high frequency coefficients of DCT and DWT are modelled by generalized Gaussian distribution. It also shows that the mid-frequency DCT coefficients can be modelled by the family of symmetric alpha-stable distributions and a detector for Cauchy distributed DCT coefficients is derived following the framework of [8]. But the both methods are based on the strong assumption that the watermark embedding strength is known to the process of watermarking detection.

Based on the above analysis, in this paper, the host image is transformed by the contourlet transform and the watermark data is embedded in the directional subband with the highest energy inspired from [9]. The contourlet sub-band detail coefficients are modelled by the distribution of generalized Gaussian density ${ }^{[8]}$.First, the host image is segmented into non-overlapping blocks and the high image energy blocks are chosen in the process of implementation. In each block, we embedded the watermark data into the contourlet coefficients. For watermark detection, the hypothesis test is utilized to detect the watermark without the original image. Finally, the detection performance of the proposed watermarking method is discussed.

\subsection{The Coutourlet Transform}

It is well known that the kernel of DWT is well suited for representing onedimensional discontinuities. However, when the dimension increases, wavelet fails to represent singularities [9]. Implementing the idea of combining subband decomposition with a directional transform, Do and Vetterli [10] introduced a multidirectional and multiscale transform known as the contourlet transform, which consists of two major stages: the subband decomposition and the directional transform. The first stage is the Laplacian Pyramid (LP) filters and Directional Filter Banks (DFB) as the second stage. The process of contourlet transform is as follows. The detail of contourlet transform can be referred to [10]. 


\subsubsection{Watermark Embedding}

The proposed watermarking algorithm performs the watermark embedding as the following steps.

(1)Segment the original image into non-overlapping $L \times L$ blocks and choose the $n$ higher ranking blocks in estimated energy measure for watermarking purposes. Then the two- pyramidal levels contourlet was applied to each selected image block.

(2)Under each selected block was transformed by the contourlet. Thus one lowpass subband image $I_{J}$ was obtained, and the band-pass directional subbands $I_{j, k}^{l_{j}}, k=1,2, \cdots 2^{l_{j}-1}, j=1,2, \cdots J$, where $j$ denotes the $j$-th level of LP decomposition, $k$ denotes $k$-th bandpass directional image decomposed by a $l_{j}$-th level DFB. It is known that the energy distribution of these directional subband images plays an important role for image processing. Therefore the average $l_{2}$ norm was used to compute the energy as follows.

$$
E_{j, k}=\frac{1}{M N} \sum_{m=1}^{M} \sum_{n=1}^{N}\left\|I_{j, k}^{l_{j}}(m, n)\right\|^{2}
$$

where $M \times N$ denotes the size of the subband image $I_{j, k}^{l_{j}}$ in each block, A larger value of the average energy of subband image implies that this subband contains more energy and should be treated as a significant subband in comparison with other subbands. As a result, in order to improve the robustness of the watermarking system, the watermark is embedded into the significant subband image with largest average energy.

(3)Use the Pseudo-random Noise generator to generate a watermark data sequence, which value is $\{-1,1\}$.

(4) Select the $N$ higher ranking blocks in estimated energy measure for embedding the watermark, the watermark embedding formula is as the following

$$
\boldsymbol{y}=\boldsymbol{x}+\lambda \cdot \boldsymbol{w}
$$

where $\lambda$ is the watermark strength factor, $\boldsymbol{x}$ represents the host image block, $\boldsymbol{y}$ is the watermarked image block. $\boldsymbol{w}$ is the watermark, the watermark length is $N_{w}$. (5)Apply the inverse contourlet transform for each selected block to obtain the watermarked image block.

(6) Combine the watermarked image blocks with the non-watermarked image blocks to get the watermarked image.

\subsubsection{Watermark Detection}

The watermark detector does not need the host image. We performed the watermark detection as the following steps. 
(1) Segment the watermarked image into $L \times L$ non-overlapping blocks. Then the two- pyramidal levels contourlet was applied to each selected block.

(2)Select the $n$ high energy blocks as the same as the watermark embedding process.

(3) With blind detection in mind, we assume that the host image acts as noise and the contourlet transform coefficients of the image are modeled by the GGD. To derive a hypothesis test, we assume that the contourlet transform coefficients represent a random sample drawn from some underlying probability density function. To drive a watermark detector, a Likelihood-Ratio Test (LRT) is constructed as the embedding function (2). We can formulate a two-sided parameter test as follows.

$$
\left\{\begin{array}{l}
H_{0}: \lambda=0, \theta(\text { no watermark }) \\
H_{1}: \lambda \neq 0, \theta(\text { watermarked })
\end{array}\right.
$$

where $\theta$ denotes the vector of unknown parameters. $H_{0}$ denotes the null hypothesis and $H_{1}$ denote the alternative hypothesis. According to noise model, $\theta$ contains the shape and scale parameter of the generalized Gaussian distribution. Given $\lambda$ and $\theta$, we can construct a Neyman-Pearson (NP) detector to decide the alternative hypothesis $H_{1}$, if

$$
\rho(y)=\frac{p\left(\boldsymbol{y} ; H_{1}\right)}{p\left(\boldsymbol{y} ; H_{0}\right)}>\mathrm{T}
$$

where $y$ denotes the received image, T denotes the detection threshold by exploiting the NP criterion. The ratio $\rho(y)$ is called the LRT.

(4) According the generalized Gaussian distribution model, we can get the LRT statistics as follows.

$$
\rho(y)=\frac{1}{c^{\beta}} \sum_{t=1}^{N}\left(|y(t)|^{\beta}-|y(t)-\lambda w(t)|^{\beta}\right), t=1,2, \cdots, N_{w}
$$

where $c, \beta$ denote the scale parameter and the shape parameter of the GGD model respectively. Using the function (5) calculate the LRT statistic, and compare the LRT with the detection threshold $\mathrm{T}$ to judge if the image was watermarked.

\section{Experimental Results}

We have performed several experiments to test the proposed method ,the 9-7 biorthogonal filters with two levels of pyramidal decomposition was utilized in the multi-scale decomposition stage and the "PKVA" filters was used for the multidirectional decomposition stage. The watermark data is embedded in the contourlet coefficients of each high energy block. 


\section{Invisibility of watermarking}

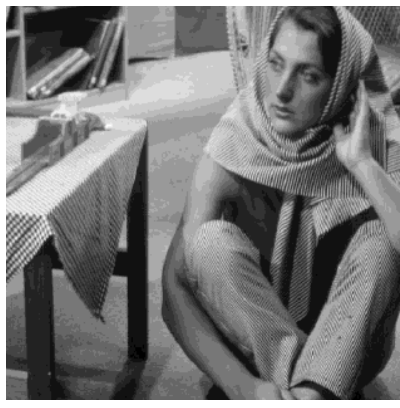

(a) Original image

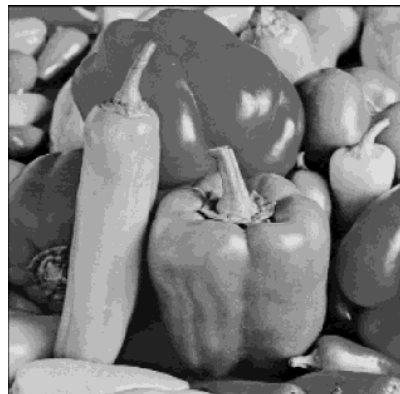

(c) Original image

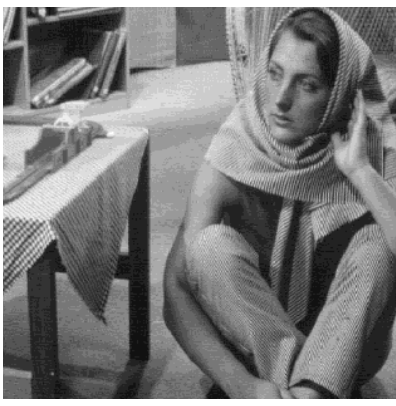

(b) Watermarked image

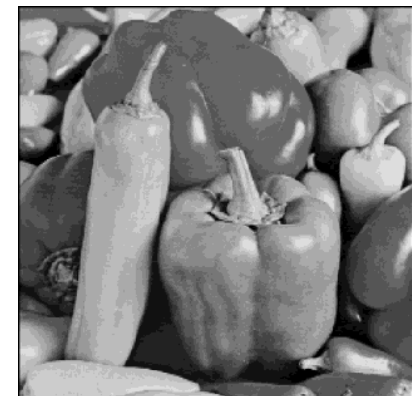

(d) Watermarked image

Fig. 1. Original test image and watermarked image by using the proposed method: Barbara Peppers.

For each image, the left is the original image, the right one is the watermarked image. In this section, we tested two standard images (Barbara and Peppes) of size $256 \times 256$. The original test images and their watermarked image version using the presented algorithm are shown in Fig.1. From the experimental tests, we can see that the watermarking scheme has good invisibility without any attacks.

\section{Robustness of watermarking}

Apart from the invisibility of watermarking, the robustness test covers common image processing and some kind of geometric distortion attacks. The watermarking scheme should be robust common image processing which could be intentional or unintentional. To evaluate the robustness of the proposed watermarking, the Bit Error Rate (BER) is applied for the performance of detection as follows.

$$
\operatorname{BER}\left(w, w^{*}\right)=\frac{\sum_{i=1}^{N_{w}} \overline{w^{*}(i) \oplus w(i)}}{N_{w}}
$$


where $w(i)$ and $w^{*}(i)$ denote the original watermark and the recovered watermark respectively, $N_{w}$ denotes the size of the watermark.

Table1.1 shows the experimental results after several attacks on the watermarked image (Barbara and Peppers) covers Gaussian Filtering, median filtering, Gaussian noise addition, JPEG compression, scaling and rotation attacks. From Table 1.1, we can see that the proposed watermarking can be robust against the common image processing and scaling and rotation attacks.

Without loss of generality, Table1.2 illustrates the comparison of proposed method with the method [11] by the BER value when against some attacks only for Barbara image, including Gaussian Filtering, median filtering, Gaussian noise addition, JPEG compression, scaling and rotation attacks. As can be see in Table 1.2 , we can see that the proposed method has superior performance than [11] when against the scaling and rotation attacks. The main reason covers two factors. The first is that we took advantages of the contourlet transform. The contoulet transform can capture much directional information of image edges. The second is that we embed the watermark information into the high energy of image blocks in the proposed watermarking method.

Table 1.1 The results under attacks

\begin{tabular}{|c|c|c|c|c|}
\hline Image & \multicolumn{2}{|c|}{ Barbara } & \multicolumn{2}{c|}{ Peppers } \\
\hline Attack & Parameters & BER(\%) & Parameters & BER(\%) \\
\hline $\begin{array}{c}\text { Gaussian Fil- } \\
\text { tering }\end{array}$ & $3 \times 3$ window & 3.85 & $3 \times 3$ window & 3.91 \\
\hline $\begin{array}{c}\text { Gaussian Fil- } \\
\text { tering }\end{array}$ & $5 \times 5$ window & 8.94 & $5 \times 5$ window & 9.19 \\
\hline $\begin{array}{c}\text { Median Fil- } \\
\text { tering }\end{array}$ & $3 \times 3$ window & 5.77 & $3 \times 3$ window & 5.34 \\
\hline $\begin{array}{c}\text { Median Filter- } \\
\text { ing }\end{array}$ & $5 \times 5$ window & 6.23 & $5 \times 5$ window & 6.78 \\
\hline Gaussian noise & Density 0.05 & 10.16 & Density 0.05 & 11.08 \\
\hline Gaussian noise & Density 0.1 & 16.29 & Density 0.1 & 17.11 \\
\hline JPEG & QF=70\% & 5.33 & QF=70\% & 5.87 \\
\hline JPEG & QF=50\% & 6.42 & QF=50\% & 6.92 \\
\hline Scaling & Factor=1.3 & 7.10 & Factor=1.3 & 7.56 \\
\hline Scaling & Factor=0.8 & 8.28 & Factor=0.8 & 8.63 \\
\hline Rotation & $10^{\circ}$ & 12.07 & 10 (Degree) & 11.90 \\
\hline Rotation & $30^{\circ}$ & 14.41 & 30 (Degree) & 15.25 \\
\hline
\end{tabular}


Table1.2 the comparison of proposed method with reference [11] by BER(\%)

\begin{tabular}{llll}
\hline Attack & Parameters & $\begin{array}{c}\text { Proposed } \\
\text { methed }\end{array}$ & Reference [1] \\
Gaussian & $5 \times 5$ window & 8.94 & 9.02 \\
Median Filtering & $5 \times 5$ window & 6.23 & 6.55 \\
Gaussian noise & Density 0.1 & 16.29 & 17.16 \\
Gaussian noise & Density 0.05 & 10.16 & 9.94 \\
JPEG & QF $=50 \%$ & 6.42 & 7.30 \\
JPEG & QF $=40 \%$ & 11.17 & 14.05 \\
Scaling & 1.3 & 7.10 & 8.53 \\
Scaling & 0.8 & 8.28 & 9.47 \\
Scaling & 0.5 & 12.34 & 15.49 \\
Rotation & $10^{\circ}$ & 12.07 & 13.01 \\
Rotation & $30^{\circ}$ & 14.41 & 17.86 \\
Rotation & $45^{\circ}$ & 20.85. & 23.70 \\
\hline
\end{tabular}

\section{Detection Performance Analysis}

Figure 2 shows the plots for the Likelihood Ratio Test detector using the method of [12]. The shape parameter $\beta$ of generalized Gaussian distribution model varies from 0.02 to 4 in Fig.2. As can be see in Fig.2, the Y-axis is the probability of false negative for the watermark detection that is also the probability of missing watermark, and the probability of false positive was given $1.0 \times$ e- 6 . For the Likelihood Ratio Test, according to the generalized Gaussian distribution model, the text arrow is the ML (Maximum likelihood) estimate. It can be see that the peak performance is missed by only a small margin from the results in Fig.2. From the results of this experimental test, it has the conclusion that the generalized Gaussian distribution model can well capture the marginal distribution of contourlet coefficients and the watermark detector has good watermark detection performance.

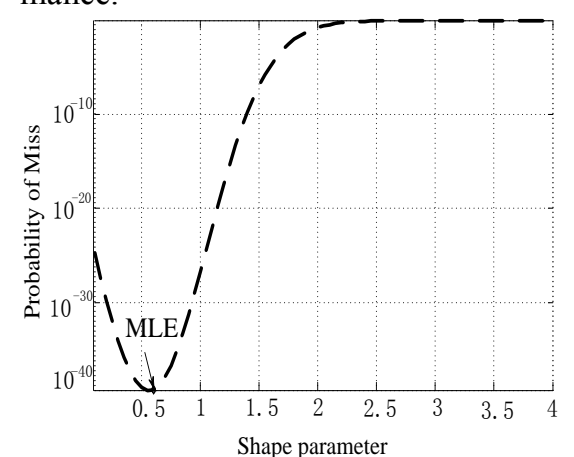

(a)

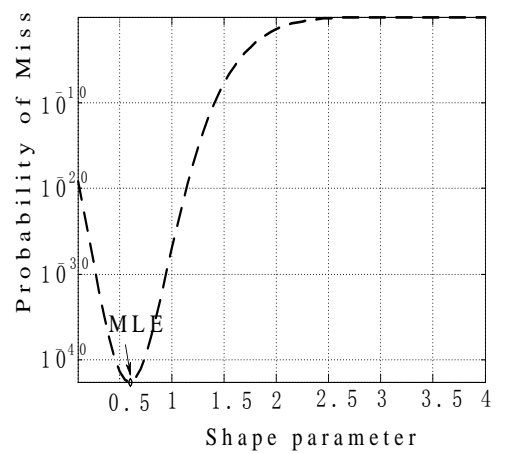

(b)

Fig.2. Detection performance of watermarking, the Likelihood Ratio test method based on Generalized Gaussian Distribution as a function of shape parameter. (a) Barbara (b) Peppers. 


\section{Conclusions}

Contourlet transform has been successfully applied in many engineering processing areas in recent years, such as image denoising, image restoration, image fusion and image watermarking et al. The contourlet transform has good perfect reconstruction, sparse representation and good directional. In this paper, we have introduced an image watermarking by using the high energy image block. This algorithm embeds the watermark by modifying the contourlet coefficients of the host image, and use the hypothesis test to develop the blind detector based on the general Gaussian distribution model. The experimental results show superior performance to common image processing attacks and some geometric attacks. Further work concentrates on developing robust watermarking scheme by integrating human visual system against geometric distortions such as cropping attack.

\subsection{References}

1. Farid.H A survey of image forgery detection. IEEE Signal Process.Mag., 2009,26(2):16-25.

2. Fabrizio G, Masahiro O Nicola A, Riccardo L.High Dynamic Range Image Watermarking Robust Against Tone-Mapping Operators. IEEE Transactions on Information Forensics and Security, 2011, 6(2):283-295.

3. Wang x, Men L.Yang h.A color image digital watermarking based on Multi-scale Features. Science in Chinese press. 2009, 39(9):966-976.

4. Cox, I., Kilian, J., Leighton, T., Shamoon, T.Secure spread spectrum watermarking for multimedia.IEEE Transactions on Image Processing, 1997, 12(6):1673-1678.

5. T. M. Ng ,H. K. Garg.Maximum-likelihood detection in DWT domain image watermarking using laplacian modeling. IEEE Signal Process. Lett., 2005,12(4): 285-288.

6. Ning B, Qi Y S, J W Huang etc. Robust Image Watermarking Based on Multiband Wavelets and Empirical Mode Decomposition. IEEE Transactions on Image Processing, 2007, 16(8): 1956-1967.

7. Kirovski.D., H.Malvar.Robust spread spectrum audio watermarking. In Proc.IEEE Int.Conf.Acoust.Speech, Signal Process.2001.1345-1348.

8. Hern'andez J. R., M. Amado, P'erez-Gonz'alez.F. DCT-domain watermarking techniques for still images: Detector performance analysis and a new structure.IEEE Transactions on Image Processing. 2000, 9(1):55-68.

9. Mohammad Ali Akhaee, S. Mohammad Ebrahim Sahraeian, Farokh Marvasti. ContourletBased Image Watermarking Using Optimum Detector in a Noisy Environment[J].IEEE Transactions Image Process. 2010,19(4):967-980.

10. M. N. Do, M. Vetterli.The contourlet transform: An efficient directional multiresolution image representation.IEEE Transactions Image Process. 2005,14(12):2091-2106.

11. Mahmood Al-khassaweneh, Selin Aviyente. Spatially Adaptive Wavelet Thresholding for Image Watermarking. IEEE International Conference on Multimedia and Expro.2006:15971600.

12. Roland Kwitt, Peter Meerwald, Andreas Uhl. Efficient Detection of Additive Watermarking in the DWT-Domain. In the Proceedings of the 17th European Signal Processing Conference in 2009, published by EURASIP. 